\title{
Zum Beitrag: Eine Kostenanalyse zur Aneuploidieuntersuchung von Eizellen im Kontext der assistierten Reproduktion in Deutschland
}

\section{Re: An Economic Analysis of Aneuploidy Screening of Oocytes in Assisted Reproduction in Germany}

Die Veröffentlichung von K. Neumann und G. Griesinger in der „GebFra“ vom Februar 2020 [1] zeigt eine Kostenkalkulation für die Anwendung von Polkörperdiagnostik (PKD) zusätzlich zu Maßnahmen der assistierten Reproduktion - und letztlich zur Vermeidung eines Aborts durch Nutzung der PKD.

Im Wesentlichen beruht das verwendete Modell auf den Zahlen der 2018 veröffentlichten „ESTEEM-Studie“ [2]. Die dort berichteten Ergebnisse sind indes kritisch zu hinterfragen. Der Anteil an befruchteten Eizellen, die nach Aneuploidietestung (Studiengruppe, 205 Patientinnen) kein Ergebnis lieferten, betrug $21 \%$ aller untersuchten Proben. Diese sehr hohe Rate ist sicher der Tatsache geschuldet, dass insgesamt 9 verschiedene Zentren mit sehr unterschiedlichen PKD-Erfahrungen an der Studie teilnahmen. In einem erfahrenen genetischen Labor mit einem hohen Probendurchsatz liegt diese Rate eher bei $6 \%$. Dadurch resultierte in der Studie auch ein hoher Anteil (33\%) an Transfers mit nicht untersuchten Embryonen in der Testgruppe. Außerdem wurden befruchtete Eizellen mit „balanciertem“ Befund (Fehler in der 1. meiotischen Teilung bei der 2. korrigiert), keinem Transfer zugeführt, obwohl sie euploid sind und somit das Potenzial für die Etablierung einer fortlaufenden Schwangerschaft haben. Aufgrund dieser Fakten wird die Wahrscheinlichkeit für eine erfolgreiche PKD sicher zu niedrig angesetzt. Trotz dieser technischen Schwächen wies die ESTEEM-Studie eine verringerte Abortrate pro „intention to treat" in der Studien- im Vergleich zur Kontrollgruppe nach (14 Aborte/64 klinischen Schwangerschaften, 21,9\% vs. 27 Aborte/72 klinischen Schwangerschaften, $37,5 \%)$.
Die vorgelegte Analyse stützt sich darüber hinaus auf Kostenangaben eines Labors für die genetische Testung mit vergleichsweise geringem Probendurchsatz. Diese Kosten bedingen den höchsten zusätzlichen zu zahlenden Anteil zur PKD für die Patienten. Neuere Analysenmethoden wie das Next Generation Sequencing sowie ein hoher Probendurchsatz verringern diese Kosten jedoch auf rund ein Drittel der hier zugrunde gelegten Preise.

In der Studie wird darüber hinaus behauptet, die Durchführung einer PKD wäre nur nach Anwendung der ICSI-Technik möglich. Dies ist definitiv unrichtig, da die Entnahme der Polkörper unter mikroskopischer Sicht erfolgt. Eventuell an die Zona pellucida gebundene und bei der Biopsie verschleppte Spermien können - anders als bei der Trophektodermbiopsie - leicht identifiziert und entfernt werden. Die Behandlung mittels Standard-IVF-Methode bedeutet also kein Hindernis für eine geplante anschließende PKD.

Auch wenn die Verhinderung von Aborten nicht primär an Geld zu messen sein kann, wäre eine Erniedrigung der Analysekosten wünschenswert, um die Methode kosteneffektiv zu gestalten und sie somit mehr Patienten anbieten zu können.

\section{Interessenkonflikt}

Das Fertility Center Hamburg bietet in Kooperation mit gametoGEN selbst Polkörperdiagnostik für Kinderwunschpatienten an.

\section{Autorinnen/Autoren}

\section{Vera Baukloh}

MVZ Fertility Center Hamburg GmbH, Hamburg

\author{
Robert Fischer \\ MVZ Fertility Center Hamburg GmbH, \\ Hamburg \\ Birthe Coburg \\ gametoGen GmbH, Hamburg
}

Susanne Knebel

gametoGen $\mathrm{GmbH}$, Hamburg

Astrid Drenckhan

gametoGen $\mathrm{GmbH}$, Hamburg

Korrespondenzadresse

Dipl.-Biol. Vera Baukloh

MVZ Fertility Center Hamburg GmbH

Speersort 4

20095 Hamburg

vera.baukloh@amedes-group.com

\section{Literatur}

[1] Neumann K, Griesinger G. An Economic Analysis of Aneuploidy Screening of Oocytes in Assisted Reproduction in Germany. Geburtsh Frauenheilk 2020; 80: 172-178. doi:10.55/a-1079-5283

[2] Verpoest W, Staessen C, Bossuyt PM et al. Preimplantation genetic testing for aneuploidy by microarray analysis of polar bodies in advanced maternal age: a randomized clinical trial. Hum Reprod 2018; 33: 17671776. doi:10.1093/humrep/dey262

\section{Publikationshinweis}

Leserbriefe stellen nicht unbedingt die Meinung von Herausgebern oder Verlag dar. Herausgeber und Verlag behalten sich vor, Leserbriefe nicht, gekürzt oder in Auszügen zu veröffentlichen.

Bibliografie

DOI https://doi.org/10.1055/a-1127-9422 Geburtsh Frauenheilk 2020; 80: 532 ๑ Georg Thieme Verlag KG Stuttgart · New York । ISSN 0016-5751 